\title{
SPATIALLY MODULATED BRAGG GRATINGS
}

\author{
I. Procházka, L. KotaČKa \\ Institute of Physical Engineering, Faculty of Mechanical Engineering \\ Technical University Brno, Technická 2, 61669 Brno, Czech Republic \\ AND F. Petráš \\ Department of Optics, Faculty of Science, Palacký University \\ tř. 17. listopadu 50, 772 07 Olomouc, Czech Republic
}

A set of coupled mode differential equations which describe the light propagation in an optical fiber containing a photoinduced Bragg grating is derived. Reflection spectra based on numerical solution to these equations are presented when basic grating parameters are varied. In addition, the influence of these parameters on the maximum reflectivity and $3 \mathrm{~dB}$ bandwidth is examined. Finally, reflection spectra of the chirped grating and the grating with spatially modulated refractive index changes are discussed.

PACS numbers: $42.70 . \mathrm{Dj}$

\section{Introduction}

Single-mode fibers have been firmly established for an efficient information transport in the telecommunications since 1950 s when the first optical fibers with a cladding layer were fabricated. In order to use the optical fibers for the future high-speed long-haul communication systems, a number of techniques (to boost the performance and overcome some detrimental limitations) has already matured (as optical fiber amplifiers based on $\mathrm{Er}^{+}$-doped fibers) or are currently pursued (such as solitons or photosensitive fibers).

Photosensitivity in Ge-doped optical fibers was reported by Hill et al. [1] for the first time. They observed the formation of a periodic permanent or quasipermanent variation of refractive index which appeared when two strong counterpropagating beams (one being launched into the fiber and the other one being reflected from the output end of the fiber) of a blue-green light interfered. The periodic variation of the refractive index formed a grating with the spatial period of one-half wavelength of the writing radiation. This procedure, however, cannot be applied to form a grating with the period around $1.55 \mu \mathrm{m}$ which is the spectral region where optical fibers exhibit the smallest absorption losses (around $3.6 \%$ of the input power per $1 \mathrm{~km}$ ). The fabrication of optical fiber gratings at longer wavelengths has become feasible only after the advent of an external writing technique 
[2] using two interfering UV beams. Moreover, when exposing an optical fiber to UV radiation a stronger and more stable modulation of the refractive index occurs. This writing technique has been further improved using the side holographic exposure with a phase mask [3]. The phase mask technique is flexible, compact, stable, and relaxes stringent requirements on spatial and temporal coherence of the writing beam.

A fiber grating can also be formed using a single high-density UV optical pulse that physically damages the fiber core $[4,5]$. Using this technique a very large index modulation can be reached. However, there are some problems with extensive short wavelength out-of-band losses due to radiation mode coupling $[4,5]$. This sort of fabrication could be well used during the process of fiber manufacturing before the fiber is coated [6].

It was also found that a hot hydrogen treatment enhances the photosensitivity of Ge-doped fibers [7]. Moreover, the use of a high-pressure (up to 800 bar) hydrogen atmosphere enables to obtain index changes greater than 0.01 [8]. Except Ge-doped fibers, strong photosensitivity has also been reported in tin-doped phosphosilicate fibers [9].

A multitude of different applications of optical fiber gratings have already emerged. They can be simply used as narrow-band reflectors (see e.g. [10, 11]). Another application concerns fiber grating semiconductor lasers [12] that allow multi-gigabit modulation with a low chirping due to the effects of the external cavity length (around $10-20 \mathrm{~mm}$ ). It is also possible to produce fiber grating lasers either by splicing the reflection grating to an Er-doped fiber [10] or by writing the grating into other rare-earth-doped fibers $[13,14]$. Chirped Bragg gratings in optical fibers may provide a tool for dispersion compensation [15, 16] or pulse compression [17]. The fiber gratings can tap the radiation out of the optical fiber to equalize the saturated gain of erbium doped fiber amplifiers within $\pm 0.3 \mathrm{~dB}[18]$. Finally, the fiber gratings can act as mode converters [19] that couple one guided mode into another one. Using fiber gratings as the mode couplers the parameters. of optical frequency filters can be greatly improved [20]. A comprehensive review of fiber gratings together with their numerous applications can be found in [21]. It is evident that the profound understanding of optical fiber gratings is very important for projection of future communication and sensorial systems.

The aim of this paper is to present a theoretical analysis of the fiber grating response when its length and refractive index changes amplitude are varied. We also show the reflection spectra of a chirped grating and demonstrate the effect of spatial modulation of refractive index variation.

\section{Coupled mode equations}

In this section we shall derive two coupled mode differential equations that describe the energy transfer between the forward and backward travelling waves.

We assume that the permittivity changes of a silica fiber are proportional to the intensity of the writing UV radiation

$$
\Delta \varepsilon(z)=\sigma I_{\mathrm{UV}}(z),
$$

where $\sigma$ is the Bragg grating encoding coefficient. As it has already been mentioned the optical fiber grating can be formed when two UV beams interfere at the fiber. 
If considering the irradiation of the fiber by two symmetric UV beams possessing a uniform intensity distribution and containing an angle $\varphi$, the interference pattern created along the fiber can be described by

$$
I_{\mathrm{UV}}(z)=2 I_{0}\left[1+\kappa \cos \left(2 k_{\mathrm{UV}} z \sin \frac{\varphi}{2}+\Phi\right)\right]
$$

where $I_{0}$ is the light intensity of interfering UV beams, respectively, $\kappa$ is a coefficient depending on the polarization properties of interfering beams, $0<\kappa \leq 1$, and $\Phi$ is a phase constant. In the special case, if both interfering UV beams are polarized perpendicularly to the incidence plane, it holds that $\kappa=1$ and Eq. (2) takes a more simple form

$$
I_{\mathrm{UV}}(z)=4 I_{0} \cos ^{2}\left(k_{\mathrm{UV}} z \sin \frac{\varphi}{2}+\frac{\Phi}{2}\right)
$$

This special case will be considered in the next treatment only.

Using Eq. (3) the optical fiber grating is described in the following way:

$$
\Delta \varepsilon(z)=\Delta \varepsilon_{\max } \sin ^{2}(\eta z)=\frac{\Delta \varepsilon_{\max }}{4}[2-\exp (\mathrm{i} 2 \eta z)-\exp (-\mathrm{i} 2 \eta z)],
$$

where $\eta=k_{\text {UV }} \sin (\varphi / 2)=2 \pi / \Lambda(\Lambda / 2$ is the actual grating period $)$ and $\Delta \varepsilon_{\max }=$ $4 \sigma I_{0}$ is the amplitude of the permittivity variation. For simplicity, a simple sine $\Delta \varepsilon$ grating is considered here, meaning that the phase in Eq. (3) was put to be $\Phi= \pm \pi$.

If we consider purely monochromatic linearly polarized light, the Helmholtz equation is

$$
\nabla^{2} E+k_{0}^{2}(\varepsilon+\Delta \varepsilon) E=0
$$

where $\varepsilon=1+\chi^{(1)}$ is the linear permittivity of the virgin medium and $k_{0}=\omega_{0} / c$. In this paper the effect of Kerr nonlinearities is ignored.

We shall consider coupling of the forward and backward propagating modes owing to the perturbation of the permittivity of the fiber core. In an isotropic medium the perturbation of $\varepsilon$ is a scalar quantity and, consequently, the only couplings TE to TE or TM to TM modes are possible but certainly the coupling TE to TM mode is forbidden [22]. For the sake of simplicity, we shall consider the coupling TE to TE modes.

The electric field vector can be expressed as a superposition of two modes propagating in the opposite direction. We suppose that the two modes are identical, only their amplitudes are different. Hence, we can write

$$
E(x, y, z)=F(x, y) A^{+}(z) \exp \left(-\mathrm{i} \beta_{0} z\right)+F(x, y) A^{-}(z) \exp \left(\mathrm{i} \beta_{0} z\right),
$$

where $A^{+}$is the amplitude of the wave that travels in the positive direction while $A^{-}$stands for the wave travelling in the opposite direction, and $\beta_{0}$ is the mode propagation constant. Both amplitudes are slowly varying functions of $z$ so it holds that

$$
\left|\frac{\partial^{2} A^{ \pm}}{\partial z^{2}}\right| \ll \beta_{0}\left|\frac{\partial A^{ \pm}}{\partial z}\right| .
$$

The coupling between the modes considered can be effective only if they are phase matched. We define the phase difference as

$$
\delta=\beta_{0}-\eta=\frac{2 \pi}{\lambda_{0}} n_{\mathrm{eff}}-\frac{2 \pi}{\Lambda},
$$


where $n_{\text {eff }}$ is the guided mode effective index. The phase matching condition is satisfied for $\beta_{0}=\eta$. Hence, if we put Eq. (6) into Eq. (5) we select only those terms which have the same $z$ phase dependence, i.e. those that contain either $\exp \left(-\mathrm{i} \beta_{0} z\right)$ or $\exp \left(\mathrm{i} \beta_{0} z\right)$ terms. The remaining terms are dropped because they describe negligible energy exchanges. In this way we arrive at two differential equations: one for the forward propagating mode and the other one for the backward propagating mode. In the following treatment we shall consider only the forward propagating mode since the equations for the backward propagating one can be derived in the same manner. After some mathematics we get two differential equations for $F(x, y)$ and $A^{+}(z)$,

$$
\frac{\partial^{2} F}{\partial x^{2}}+\frac{\partial^{2} F}{\partial y^{2}}+\left[k_{0}^{2}\left(\varepsilon+\frac{\Delta \varepsilon_{\max }}{2}\right)-\bar{\beta}^{2}\right] F=0
$$

and

$$
2 \mathrm{i} \beta_{0} \frac{\partial A^{+}}{\partial z}-\left(\bar{\beta}^{2}-\beta_{0}^{2}\right) A^{+}=-\frac{k_{0}^{2} \Delta \varepsilon_{\max }}{4} A^{-} \exp (\mathrm{i} 2 \delta z),
$$

where we have neglected, with the respect to the condition (7), the second derivative of $A^{+}$.

The permittivity changes are related to the refractive index changes in the following way:

$$
n+\Delta n_{\max }=\left(\varepsilon+\Delta \varepsilon_{\max }\right)^{1 / 2} \approx \varepsilon^{1 / 2}+\frac{\Delta \varepsilon_{\max }}{2 \varepsilon^{1 / 2}} .
$$

During the grating fabrication we can determine the amplitude of the refractive index changes $\Delta n_{\max }$, which is assumed to be small $\left(\Delta n_{\max } \ll n\right)$. Hence, the amplitude of the permittivity variation can be expressed as

$$
\Delta \varepsilon_{\max } \approx 2 n \Delta n_{\max } \text {. }
$$

Equation (9) can be solved using the first-order perturbation theory [23]. In the first order $\Delta n_{\max }$ does not affect the modal distribution $F(x, y)$ but is related to the eigenvalue $\bar{\beta}$, which can be determined by means of Eq. (9) as follows [24], as

$$
\bar{\beta}=\beta_{0}+\Delta \beta
$$

where

$$
\Delta \beta=k_{0} \frac{\iint_{-\infty}^{\infty} \Delta n|F(x, y)|^{2} \mathrm{~d} x \mathrm{~d} y}{\iint_{-\infty}^{\infty}|F(x, y)|^{2} \mathrm{~d} x \mathrm{~d} y}=k_{0} \Delta n \Gamma .
$$

Here $\Gamma$ is the mode confinement factor defined as [25]

$$
\Gamma=\frac{\iint_{\text {core }}|F(x, y)|^{2} \mathrm{~d} x \mathrm{~d} y}{\iint_{-\infty}^{\infty}|F(x, y)|^{2} \mathrm{~d} x \mathrm{~d} y}
$$

The integration in the numerator on the right hand side of Eq. (15) is to be taken over the core cross-section area because $\Delta n_{\max }$ is zero in the cladding region. This assumption is based on the fact that the refractive index perturbation exists only in the fiber core since it is doped with germanium.

We are now ready to write coupled mode differential equations for the complex field amplitudes of propagating modes. If we approximate $\bar{\beta}^{2}-\beta_{0}^{2}$ in Eq. (10) 
by $2 \beta_{0}\left(\bar{\beta}-\beta_{0}\right)=2 \beta_{0} \Delta \beta$ and perform the same procedure for the backward propagating mode we get

$$
\begin{aligned}
& \frac{\partial A^{+}}{\partial z}=-\mathrm{i} \Delta \beta A^{+}+\mathrm{i} K A^{-} \exp (\mathrm{i} \phi) \\
& \frac{\partial A^{-}}{\partial z}=\mathrm{i} \Delta \beta A^{-}-\mathrm{i} K A^{+} \exp (-\mathrm{i} \phi)
\end{aligned}
$$

where the coupling coefficient $K$ is defined as

$$
K=\frac{k_{0}^{2} \Delta \varepsilon_{\max }}{8 \beta_{0}} \approx \frac{k_{0} n \Delta n_{\max }}{4 n_{\mathrm{eff}}} .
$$

Equations (16) govern the propagation of monochromatic waves through the fiber grating. Depending on the phase difference $\delta$ we observe the drop in the forward propagating mode which is accompanied by the concurrent transfer of the energy to the backward propagating one.

The Bragg grating can also be described as a sequence of $\exp \left(-\gamma z^{2}\right)$ profiles truncated in such a way that the grating would have the required period. If we expand the function $\exp \left[-\gamma(z-\Lambda / 4)^{2}\right]$ into the Fourier series within the interval $\langle 0, \Lambda / 2\rangle$

$$
\exp \left[-\gamma(z-\Lambda / 4)^{2}\right]=\sum_{n} c_{n} \exp (\mathrm{i} 4 \pi n z / \Lambda)
$$

where

$$
c_{n}=\frac{2}{\Lambda} \int_{0}^{\Lambda / 2} \exp \left[-\gamma(z-\Lambda / 4)^{2}\right] \exp (-\mathrm{i} 4 \pi n z / \Lambda) \mathrm{d} z
$$

then the coefficients $\Delta \beta$ and $K$ on the right hand side of Eqs. (16) are to be multiplied by $2 c_{0}$ and $4 c_{1}$ respectively. This procedure can be, in general, applied to any grating profile.

Finally, we introduce one parameter - the grating strength $K L$ where $L$ is the grating length. The grating strength is the dimensionless product which pertains to the maximum reflectivity. We notice that after the substitution $K=$ $\psi K^{\prime}$ and $L=L^{\prime} / \psi$, where $\psi$ is an arbitrary constant, the grating strength remains unchanged. Hence, once we know the maximum reflectivity for a particular value of $\Delta n_{\max }$ and $L$ we immediately know it for other values of these parameters which can be derived using the above substitution.

In the next sections we present our results of the numerical solution to the coupled mode differential equations (16) under various conditions.

In order to compute reflection spectra we have used the Runge-Kutta method of the third-order subject to the following boundary conditions for the normalized complex field amplitudes:

$$
A^{+}(0)=1, \quad A^{-}(L)=0 .
$$

The reflectivity and transmissivity are defined as

$$
R=\left|A^{-}(0)\right|^{2}, \quad T=\left|A^{+}(L)\right|^{2} .
$$


We shall demonstrate how two important characteristics namely the maximum reflectivity $R_{\max }$ and the $3 \mathrm{~dB}$ bandwidth depend on the basic fiber grating parameters: the amplitude of refractive index changes $\Delta n_{\max }$ and the grating length $L$. We also present reflection spectra when the refractive index variation amplitude $\Delta n_{\max }$ is modulated by the function $\exp \left(-\alpha z^{2}\right)$ and when the grating is chirped.

The following parameters of a step-index single-mode fiber were used for the computation: the refractive index of the core $n_{1}=1.447$ and of the cladding $n_{2}=1.442$; the radius of the core for the single-mode operation at the input wavelength $\lambda=1.55 \mu \mathrm{m}$ was taken $a=4.7 \mu \mathrm{m}$. The Bragg wavelength was computed to be $\Lambda=1.073 \mu \mathrm{m}$.

\section{Ordinary grating}

We start with varying the grating length $L$ and keeping the amplitude of refractive index changes $\Delta n_{\max }$ constant. Increasing $L$ we increase the grating strength $K L$ and, hence, the coupling becomes more efficient. The reflection spectra for three various grating lengths as functions of the difference $\delta \lambda=\lambda-\lambda_{0} / n_{\text {eff }}$ are presented in Fig. 1. Not only does the maximum reflectivity increase with increasing $L$ but the sidelobes do so as well. We observe that the peak reflectivity does not occur for $\delta \lambda=0$. There is the following reason for that. The reflection wavelength can be calculated from the Bragg condition

$$
\lambda_{\mathrm{Bragg}}=\Lambda n_{\mathrm{eff}},
$$

i.e. when considering zero phase difference $\delta$ in Eq. (8). Because the introduced refractive index changes are only positive (the refractive index increases with intensity of UV writing radiation) the light "sees" higher refractive index in the grating compared with its virgin parts. Therefore, the maximum reflectivity occurs for red shifted wavelength $\lambda_{M}$ :

$$
\lambda_{M}=\Lambda\left(n_{\mathrm{eff}}+\frac{\Delta n_{\max }}{2} \Gamma\right) .
$$

We see that the Bragg condition depends upon the UV-intensity via the refractive index variation amplitude $\Delta n_{\max }$. At $\lambda_{M}$ the reflected light is all in phase. For the wavelengths being far from $\lambda_{M}$ the reflected light is out of phase so that the light propagates throughout the grating withont any reflection.

The maximum reflectivity $R_{\max }$ and the $3 \mathrm{~dB}$ bandwidth as functions of $L$ are depicted in Figs. 2 and 3, respectively, for three different refractive index changes amplitudes. Both the quantities saturate for large values of $L$. The saturation value of $R_{\max }$ is one and the saturation value of the $3 \mathrm{~dB}$ bandwidth depends on $\Delta n_{\max }$. The greater the value of $\Delta n_{\max }$ the more quickly they saturate.

As the next step we vary the amplitude of refractive index changes $\Delta n_{\max }$ while. $L$ is kept constant. The reflection spectra for three different amplitudes $\Delta n_{\max }$ are shown in Fig. 4 that well illustrates the red-shift of $\lambda_{M}$. Looking at Figs. 5 and 6 we find similar behaviour to the previous case: the grating strength and the maximum reflectivity $R_{\max }$ increase but the $3 \mathrm{~dB}$ bandwidth increases in this case as well. At larger $\Delta n_{\max }$ the reflection spectra have a flat close-to-one 


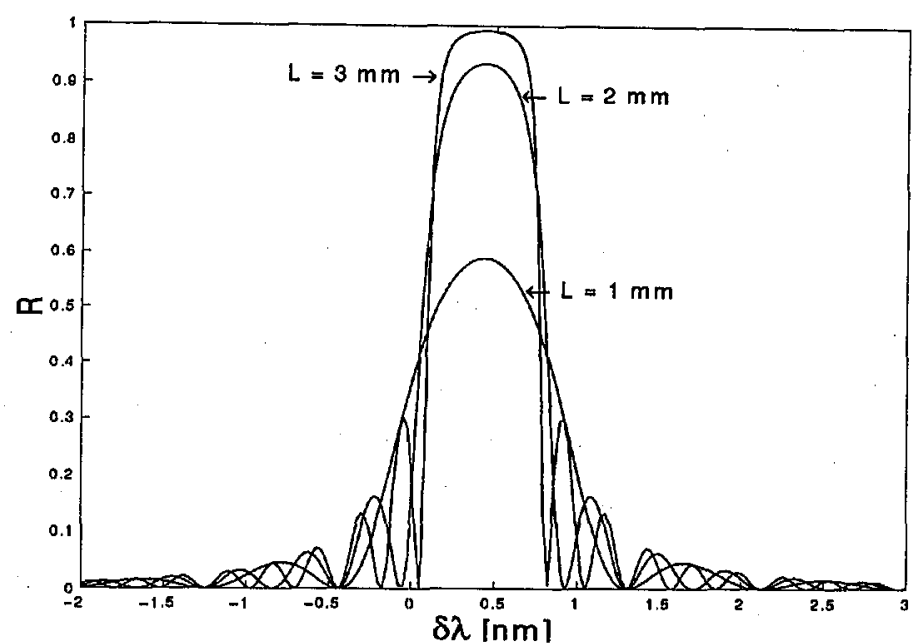

Fig. 1. The reflection spectra for different lengths of the uniform Bragg grating for $\Delta n_{\max }=0.001$.

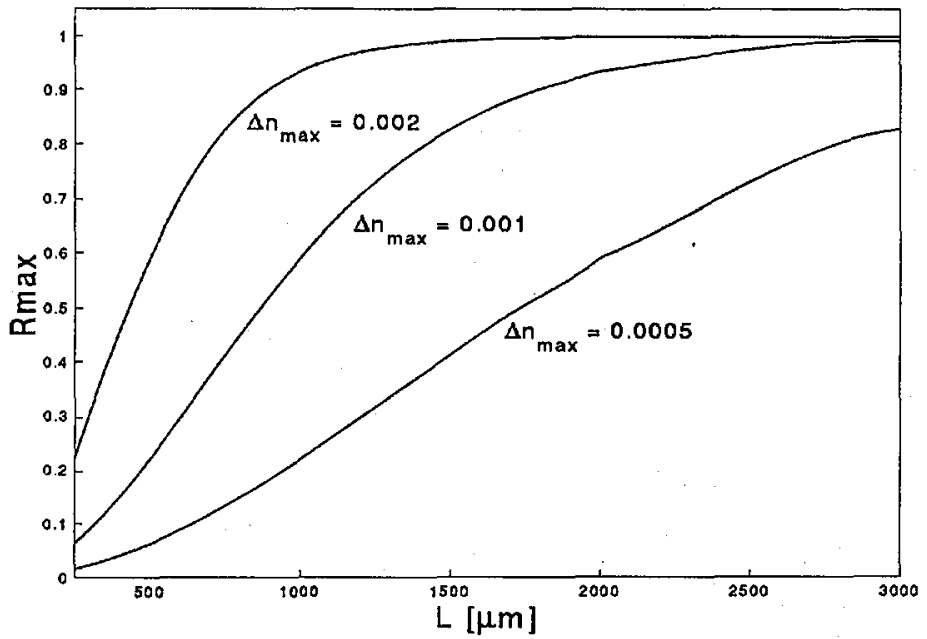

Fig. 2. The maximum reflectivity as a function of the grating length $L$ for different . values of $\Delta n_{\max }$.

reflectivity for a fairly large interval of wavelengths while the sidelobes are more pronounced.

As can be seen from Fig. 4, the reflectivity at a given wavelength (e.g. the wavelength corresponding to $\delta \lambda \approx 2 \mathrm{~nm}$ ) depends on the value of $\Delta n_{\max }$. The light at this given wavelength is only slightly reflected for $\Delta n_{\max }=0.001$. Increasing the value of $\Delta n_{\max }$ to 0.005 we achieve almost $100 \%$ reflectivity whereas decreasing the value of $\Delta n_{\max }$ we scarcely obtain any reflection. The propagation of optical pulses would complicate the situation since the refractive index (as a function 


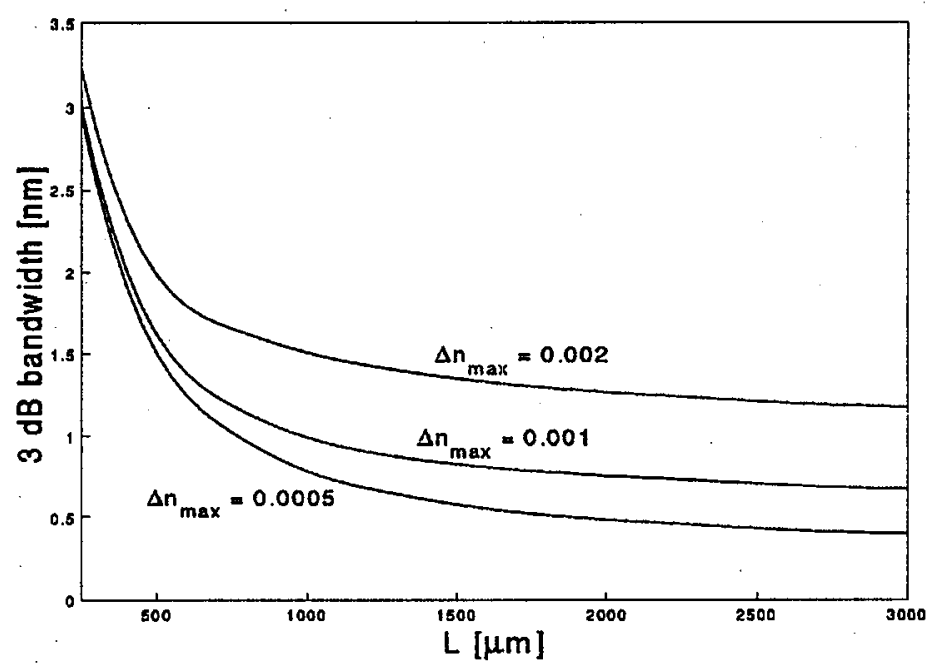

Fig. 3. The $3 \mathrm{~dB}$ bandwidth as a function of the grating length $L$ for different values of $\Delta n_{\max }$.

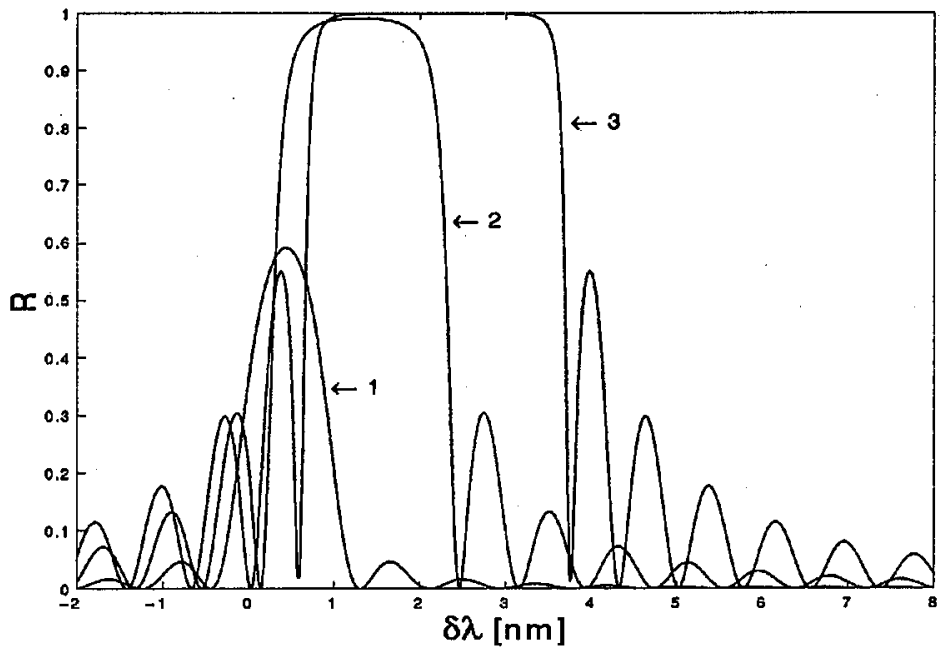

Fig. 4. The reflection spectra for different refractive index changes amplitudes $\Delta n_{\max }$ of the uniform Bragg grating for $L=1000 \mu \mathrm{m}$.

of pulse intensity, see [24]) increases in the center of the pulse due to the Kerr nonlinearity. In addition, the new phenomena like gap solitons may appear [26].

The following conclusions are obvious. If we are interested in very small $3 \mathrm{~dB}$ bandwidths we should work with long gratings possessing small refractive index changes. On the other hand, to design a grating with a broad band reflectivity it is necessary to increase $\Delta n_{\max }$ according to the desired bandwidth.

In the conclusion of this section we would like to stress that if the refractive index perturbation was modelled using just $\sin (\eta z)$ instead of $\sin ^{2}(\eta z)$ grating 


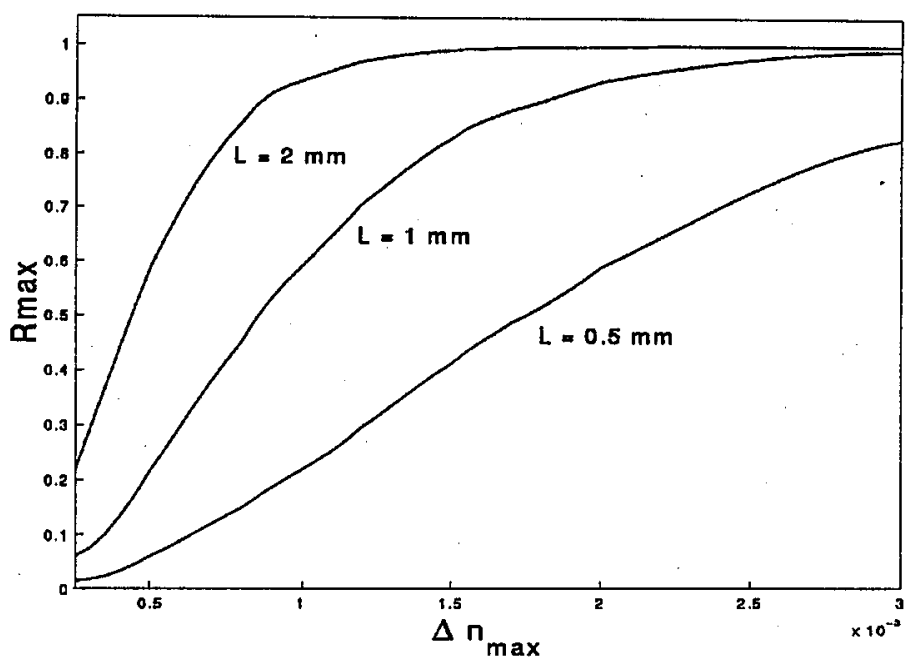

Fig. 5. The maximum reflectivity as a function of the refractive index changes amplitude $\Delta n_{\max }$ for different grating lengths.

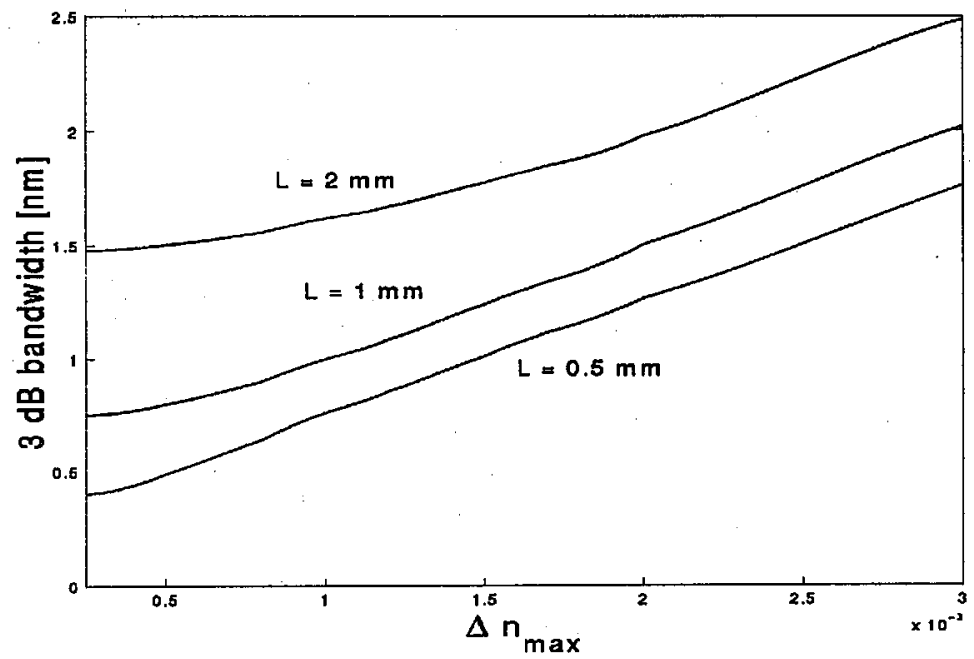

Fig. 6. The $3 \mathrm{~dB}$ bandwidth as a functions of the refractive index changes amplitude $\Delta n_{\max }$ for different grating lengths.

profile then the maximum reflectivity would appear at $\delta \lambda=0$ for any value of $\Delta n_{\max }$ so that the Bragg condition would be independent of $\Delta n_{\max }$. However, $\sin (\eta z)$ is not the appropriate function because it does not realistically describe the grating formation. The light intensity is always positive and does not take negative values as $\sin (\eta z)$ does. The function $\sin ^{2}(\eta z)$ describes the one-photon absorption which is responsible for the grating formation if using UV radiation. If we use blue-green light then we have to deal with two-photon absorption, which is a much weaker process, and the $\sin ^{2}(\eta z)$ should be replaced by $\sin ^{4}(\eta z)$. 


\section{Spatially modulated grating}

It is well known from the theory of coherence of light that the interference pattern is spatially modulated depending on the spatial coherence degree (see e.g. $[27,28])$. We suppose, for the sake of simplicity, that the interference pattern is given by the function

$$
I \sim I_{0} \cos ^{2}(2 \eta z) \exp \left[-\alpha\left(\frac{z-L / 2}{L}\right)^{2}\right],
$$

where the Gaussian function $M(z)=\exp \left[-\alpha(z-L / 2)^{2} / L^{2}\right]$ describes the spatial modulation of the interference pattern. The parameter $\alpha$ pertains to the strength of interference fringes modulation.

In the next treatment we shall compute the response of the grating whose amplitude of refractive index changes is spatially modulated. Thus, the right hand sides of Eqs. (16) are to be multiplied by the function $M(z)$. According to the above definition the strongest modulation occurs at both ends of the grating whereas it is equal to zero at the grating center. Two reflection spectra are shown in Fig. 7. The spectrum with the highest $R_{\max }$ pertains to the index grating without any. modulation and is to be compared with the other two ones which present the response of the modulated index grating (i.e. for $\alpha>0$ ). Increasing $\alpha$ we decrease the maximum reflectivity because the interaction length for any wavelength gets smaller. At the same time the position of $\lambda_{M}$ slightly moves to the left because the applied modulation affects the mean value of the refractive index changes, which should otherwise be $\Gamma \Delta n_{\max } / 2$. The $3 \mathrm{~dB}$ bandwidth first narrows a bit but then monotonously increases. The results are summarized in Figs. 8 to 10 .

It was shown [29] that for the frequencies smaller than the bandgap frequencies the grating modulated with a Gaussian profile behaves like a Fabry-Perot.

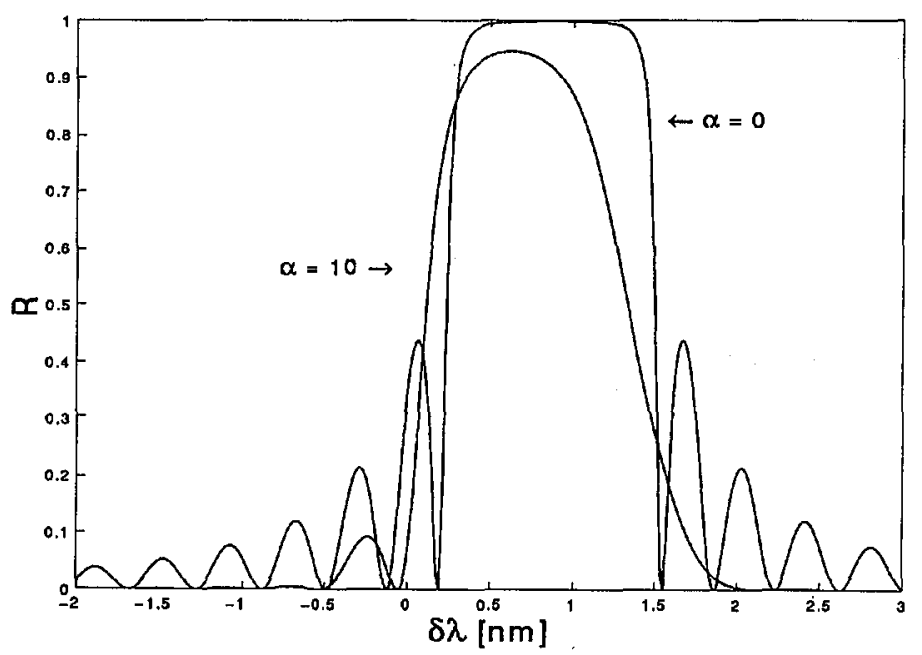

Fig. 7. The reflection spectra for different values of the modulation parameter $\alpha(L=$ $2000 \mu \mathrm{m}$ and $\Delta n_{\max }=0.001$ ). 


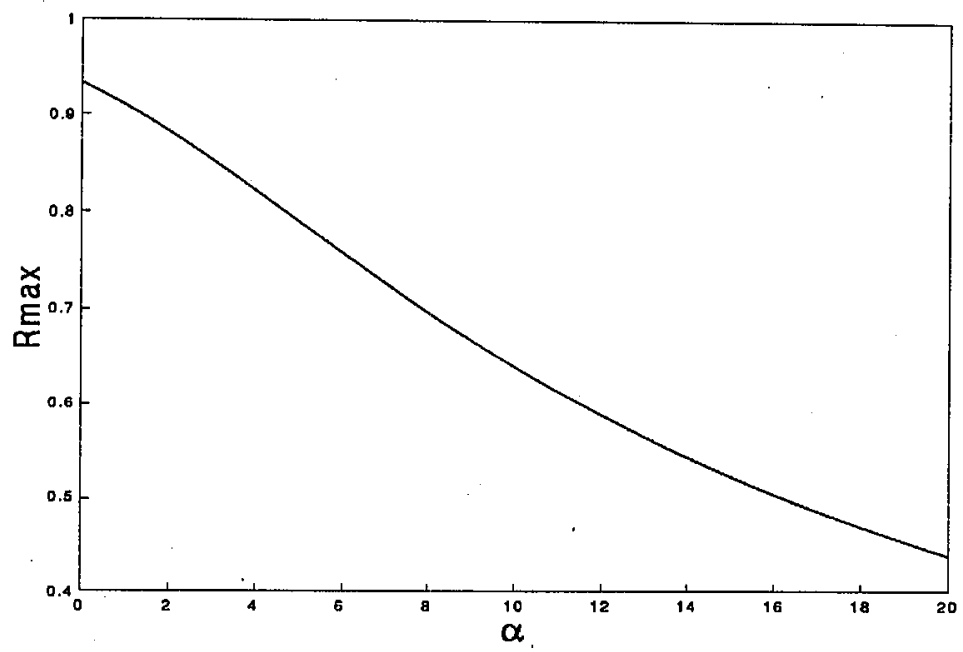

Fig. 8. The maximum reflectivity as a function of the modulation parameter $\alpha$ ( $L=$ $2000 \mu \mathrm{m}$ and $\Delta n_{\max }=0.001$ ).

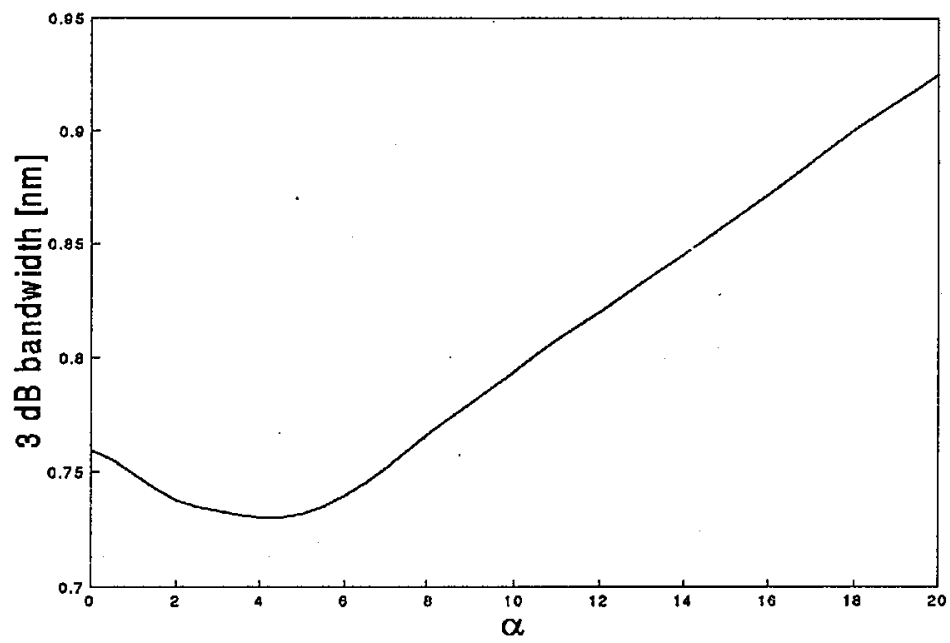

Fig. 9. The $3 \mathrm{~dB}$ bandwidth as functions of the modulation parameter $\alpha(L=2000 \mu \mathrm{m}$. and $\Delta n_{\max }=0.001$ ).

interferometer. Hence, the interference maxima, when condition for constructive interference is satisfied, appear at the left hand side of the reflection spectra.

Chirped gratings have become one of the means how to suppress the crippling effect of dispersion in optical fibers $[15,16]$. In order to introduce the grating chirp we modify the phase difference in the following way:

$$
\phi=2 \int_{0}^{z} \delta\left(z^{\prime}\right) \mathrm{d} z^{\prime}=\left(\frac{4 \pi}{\lambda_{0}} n_{\mathrm{eff}}-\frac{4 \pi}{\Lambda}\right) z-\frac{4 \pi}{\Lambda} \frac{C}{2} z^{2},
$$




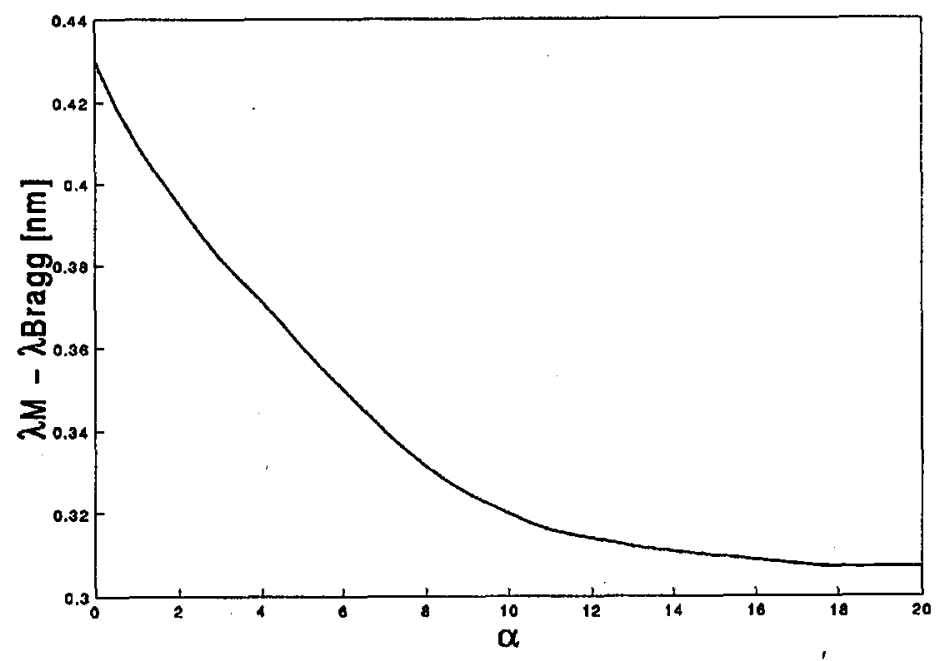

Fig. 10. The value of the difference $\lambda_{M}-\lambda_{\text {Bragg }}$ as a function of the modulation parameter $\alpha\left(L=2000 \mu \mathrm{m}\right.$ and $\left.\Delta n_{\max }=0.001\right)$.

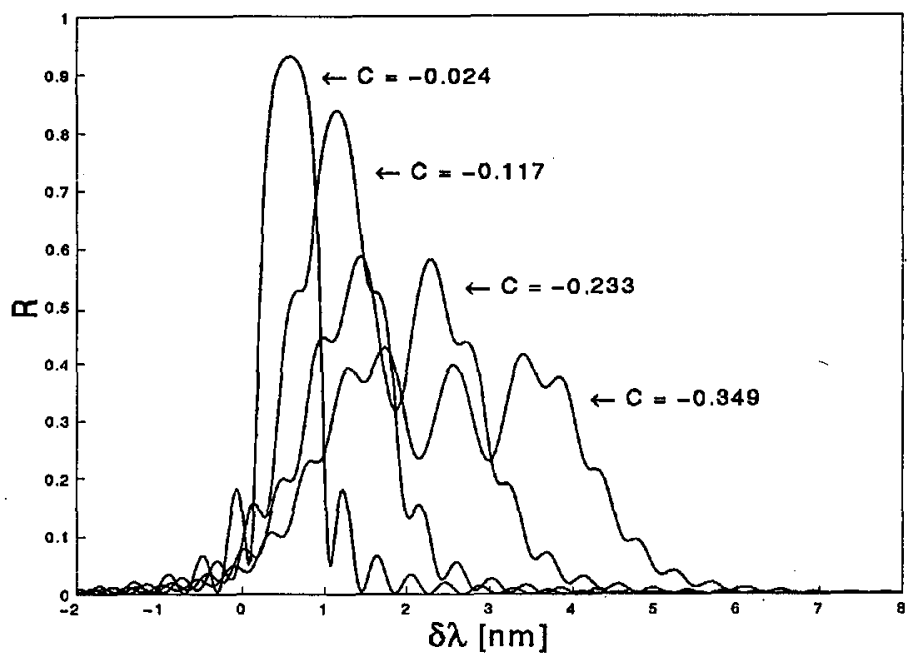

Fig. 11. The reflection spectra for different chirp parameters $C<0(L=.2000 \mu \mathrm{m}$, $\Delta n_{\max }=0.001$, and $\left.\alpha=0\right)$.

where $C\left[\mathrm{~nm}^{-1}\right]$ is the chirp parameter. Varying $C$ we get the spectra displayed in Fig. 11 (for positive values of $C$ the spectra are identical but blue-shifted). If $C$ is negative $A$ increases and vice versa. Increasing $|C|$ the grating bandwidth increases. It is obvious that due to the smaller interaction length at any wavelength the maximum reflectivity drops. The $3 \mathrm{~dB}$ bandwidth increases due to the chirp.

The chirped grating can provide another way how to form a broadband filter. Simply by increasing $\Delta n_{\max }$ we compensate for the lower reflectivity. We have found that for $C=-0.233$ and $\Delta n_{\max }=0.003$ the maximum reflectivity 


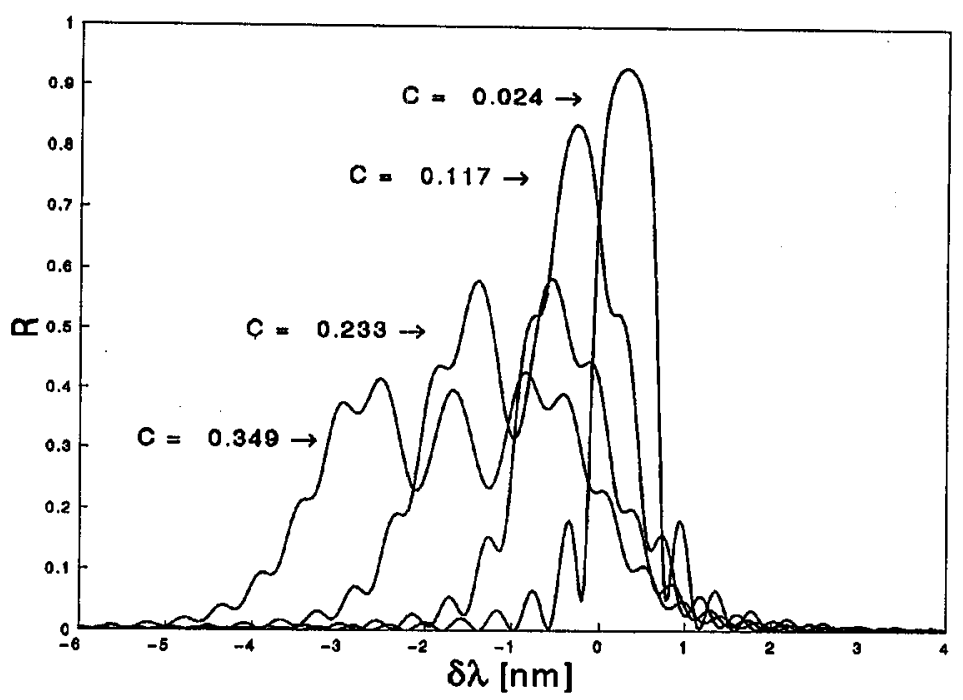

Fig. 12. The reflection spectra for different chirp parameters $C>0(L=2000 \mu \mathrm{m}$, $\Delta n_{\max }=0.001$, and $\alpha=0$ ).

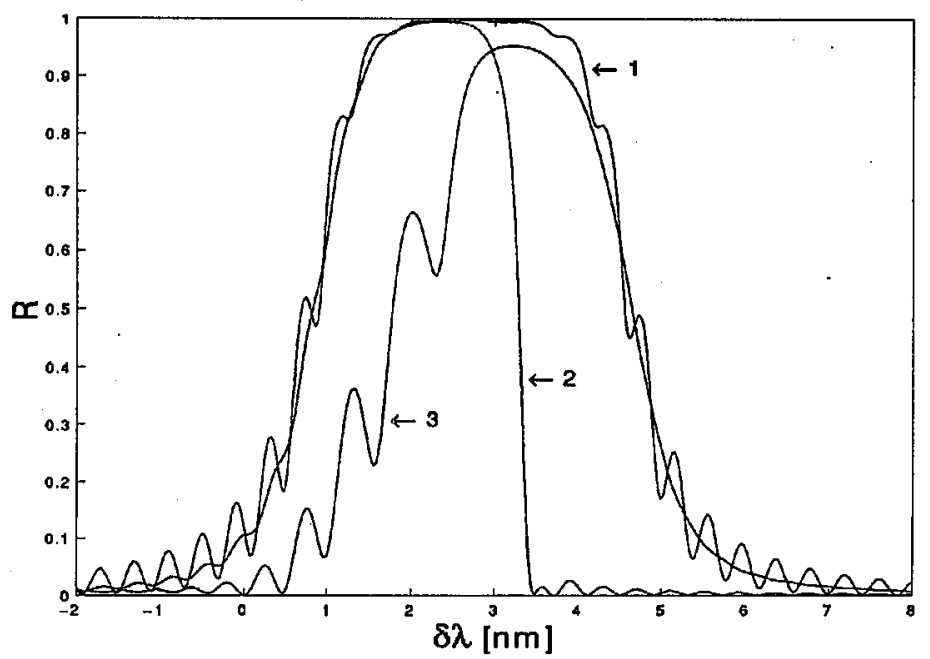

Fig. 13. The reflection spectra of a chirped grating: $L=2000 \mu \mathrm{m}, \Delta n_{\max }=0.003$, and $C=-0.233$. The spectrum 1 is for $\alpha=0$, the spectrum 2 is for $\alpha=4$ and $M(z)$ positioned at $z=0.3 L$, and the spectrum 3 is for $\alpha=4$ and $M(z)$ positioned at $z=0.9 L$.

$R_{\max }$ approaches one and $3 \mathrm{~dB}$ bandwidth increases to $\approx 3.7 \mathrm{~nm}$ compared to the uniform grating for which the $3 \mathrm{~dB}$ bandwidth is $\approx 2.5 \mathrm{~nm}$. For determining $\lambda_{M}$ both the chirp and the value of $\Delta n_{\max }$ have to be combined. The spatial modulation further complicates the situation. Increasing $\alpha$ the $3 \mathrm{~dB}$ bandwidth decreases (for $\alpha=4$ down to $\approx 3 \mathrm{~nm}$ ). Numerical calculations show that the position of the maximum of $M(z)$ also plays an important role. If the maximum of 
$M(z)$ is closer to the input end of the fiber grating $(z=0) \lambda_{M}$ gets blue-shifted and $3 \mathrm{~dB}$ bandwidth is further reduced. Moreover, the spectrum is distinctly smoother and without sidelobes. If the maximum of $M(z)$ is closer to the output end of the fiber grating $(z=L)$ the reflection peak becomes distorted. By moving the profile $M(z)$ within the grating we can select those parts of the grating that significantly reflect light and suppress the unwanted ones. We have found that for $\alpha>0$ the spectra become noticeably asymmetric. The asymmetry contradicts the numerical calculation in [30] but it is in the agreement with the measured data in [16]. The above discussion is summarized in Fig. 12. The grating parameters are: $L=2000 \mu \mathrm{m}, \Delta n_{\max }=0.003$, and $C=-0.233$. The reflection spectra (Fig. 13) correspond to $\alpha=0, \alpha=4$ with the $M(z)$ maximum positioned at $z=0.3 L$, and $\alpha=4$ with the maximum of $M(z)$ positioned at $z=0.9 L$.

\section{Conclusion}

We have derived the set of coupled mode differential equations that describe the coupling of the forward mode to the backward one in an optical fiber due to the presence of the periodic refractive index changes. The procedure was based on the solution to the nonlinear wave equation for the optical fiber. The refractive index changes were treated as a small perturbation. The coupled mode equations were solved numerically and the results were presented in the form of several plots. First, the grating length and the amplitude of perturbation were varied and their influence on the maximum reflectivity and $3 \mathrm{~dB}$ bandwidth were demonstrated. It has been found that the increase in the grating length enhances the maximum reflectivity, whilst the increase in the refractive index changes amplitude not only enhances the maximum reflectivity but it also causes a red shift of the reflectivity peak. The $3 \mathrm{~dB}$ bandwidth drops with the increasing grating length and it saturates at a value that depends on the perturbation amplitude. On the other hand, the increase in the grating amplitude increases the bandwidth. Second, both the fiber grating period and the refractive index changes amplitude were spatially modulated. It has shown that the spatial modulation of the grating amplitude suppresses the occurrence of sidelobes which is accompanied with the drop in the maximum reflectivity. When the fiber grating is chirped then different parts of the grating reflects the light at different wavelengths. This results in the drop of the maximum reflectivity and the reflection spectra become broadened.

\section{Acknowledgments}

The authors would like to thank Prof. P. Chmela for useful discussion and assistance.

\section{References}

[1] K.O. Hill, Y. Fujii, D.C. Johnson, B.S. Kawasaki, Appl. Phys. Lett. 32, 647 (1978).

[2] G. Meltz, W.W. Morey, W.H. Glenn, Opt. Lett. 14, 823 (1989).

[3] K.O. Hill, B. Mallo, F. Bilodeau, D.C. Johnson, J. Albert, Appl. Phys. Lett. 62, 1035 (1993).

[4] J.-L. Archambault, L. Reekie, P.St.J. Russell, Electron. Lett. 29, 453 (1993). 
[5] B. Malo, D.C. Johnson, F. Bilodeau, J. Albert, K.O. Hill, Opt. Lett. 18, 1277 (1993).

[6] L. Dong, J.-L. Archambault, L. Reekie, P.St.J. Russell, D.N. Payne, Electron. Lett. 29, 1577 (1993).

[7] F. Ouellette, K.O. Hill, D.C. Johnson, Appl. Phys. Lett. 54, 1086 (1989).

[8] P. Lemaire, R.M. Atkins, V. Mizrahi, W.A. Reed, Electron. Lett. 29, 136 (1993).

[9] L. Dong, J.L. Cruz, J.A. Tucknott, L. Reekie, D.N. Payne, Opt. Lett. 20, 1982 (1995).

[10] R. Kashyap, J.R. Armitage, R. Wyatt, S.T. Davey, D.L. Williams, Electron. Lett. 26, 730 (1990).

[11] J.-M.P. Delavaux, C.R. Giles, S.W. Granlund, C.D. Chen, Opt. Fiber Technol. 2, 351 (1996).

[12] D.M. Bird, J.R. Armitage, R. Kashyap, R.M.A. Fatah, K.H. Cameron, Electron. Lett. 27, 1115 (1991).

[13] G.A. Ball, W.W. Morey, J.P. Waters, Electron. Lett. 26, 1829 (1990).

[14] J.T. Kringlebotn, J.-R. Archambault, L. Reekie, R.I. Laming, D.N. Payne, Opt. Fiber Technol. 2, 69 (1996).

[15] H.G. Winful, Appl. Phys. Lett. 46, 975 (1993).

[16] S. Barcelos, M.N. Zervas, R.I. Laming, Opt. Fiber Technol. 2, 213 (1996).

[17] R. Kashyap, S.V. Chernikov, P.F. McKee, J.R. Taylor, Electron. Lett. 30, 1078 (1994).

[18] R. Kashyap, R. Wyatt, P.F. McKee, Electron. Lett. 29, 1025 (1993).

[19] F. Bilodeau, K.O. Hill, B. Malo, D.C. Johnson, I.M. Skinner, Electron. Lett. 27, 1614 (1990).

[20] C.-X. Shi, IEEE J. Quantum Electron. 32, 1360 (1996).

[21] R. Kashyap, Opt. Fiber Technol. 1, 17 (1994).

[22] A. Yariv, Quantum Electronics, Wiley, New York 1989, p. 609.

[23] P.M. More, H. Freshbach, Methods of Theoretical Physics, McGraw-Hill, New York 1953, Ch. 9.

[24] G.P. Agrawal, Nonlinear Fiber Optics, Academic Press, Boston 1989, Ch. 2.

[25] I.P. Kaminow, R.S. Tucker, in: Guided-Wave Optoelectronics, Ed. T. Tamir, Springer-Verlag, New York 1990, p. 220.

[26] C.M. de Sterke, J.E. Sipe, in: Progress in Optics $X X X I I$, Ed. E. Wolf, Elsevier, Amsterdam 1997, p. 205.

[27] B.E.A. Saleh, M.C. Teich, Fundamentals of Photonics, Wiley, New York 1991, Ch. 10.

[28] M. Born, E. Wolf, Principles of Optics, Pergamon Press, New York 1993, Ch. 10.

[29] J.E. Sipe, L. Poladian, C.M. de Sterke, J. Opt. Soc. Am. A 11, 1307 (1994).

[30] S. Thibault, J. Lauzon, J.-F. Cliche, J. Martin, M.A. Duguay, M. Têtu, Opt. Lett. 20, 647 (1995). 